\title{
À propos de quelques exceptions aux règles de la liaison et de l'élision
}

\author{
Lotfi Abouda, Céline Dugua, Guillaume Enguehard \\ LLL, UMR 7270, Université d'Orléans
}

\begin{abstract}
Résumé. La liaison et l'élision en français sont deux phénomènes phonologiques largement décrits en phonologie, et, plus largement, par les grammairiens, les sociolinguistes, les chercheurs en acquisition, etc. Ces phénomènes peuvent être traités simultanément puisque tous deux correspondent à une modification phonologique à la frontière entre deux mots en raison des propriétés des mots en question et de leur relation syntaxique. Dans cet article, nous nous proposons de présenter quelques cas particuliers de non liaison ou de non élision connus pour certains, et peu ou pas abordés dans la littérature, pour d'autres. Citons par exemple l'absence possible d'élision devant les lettres (ex: le L majuscule) ou devant les chiffres (ex : un onze de France), devant certaines couleurs (ex : le 'orange de ta veste), devant des noms propres (ex: le Onfray d'aujourd'hui), et devant des sigles (le FMI). La phonologie ne traite pas ces derniers cas. En faisant appel à des traitements sémantico-syntaxiques, nous proposons que la présence d'une position syntaxique vide entre les deux mots (la [couleur] orange de ta veste) bloque la réalisation de la liaison ou de l'élision. L'absence de la liaison ou de l'élision au lieu d'être vue comme une exception inexplicable à un phénomène attendu, ne serait que la manifestation en phonologie d'une barrière syntaxique séparant des éléments linéairement contigus. Cette hypothèse largement illustrée dans l'article permet de faire se rejoindre phonologie et syntaxe.
\end{abstract}

\begin{abstract}
About some exceptions to the rules of liaison and elision Liaison and elision in French are two phonological phenomena widely described in phonology and, more broadly, by grammarians, sociolinguists, acquisition researchers, etc. These phenomena can be treated simultaneously since both correspond to a phonological modification at the boundary between two words due to their properties and their syntactic relationship. In this paper, we propose to present some special cases of non-liaison or non-elision known or no literature coverage for others. Let us quote for example the possible absence of elision in front of letters (e.g. le L majuscule) or numbers (e.g. un onze de France), in front of certain colors (e.g. the orange of your jacket), in front of proper names (e.g. le Onfray d'aujourd'hui), and in front of acronyms (e.g. le FMI). Phonology does not deal with these last cases. Using semantic-syntactic treatments, we propose that the presence of an empty syntactic position between the two words (la [couleur] orange de ta veste) blocks the realization of the liaison or the elision. The absence of liaison or elision, instead of being seen as an inexplicable exception to an expected phenomenon, would be merely the manifestation in phonology of a syntactic barrier separating
\end{abstract}


linearly contiguous elements. This hypothesis, widely illustrated in the article, allows us to bring phonology and syntax together.

\section{Introduction $^{\mathrm{i}}$}

La liaison et l'élision sont deux phénomènes bien connus du français, conditionnés premièrement par la nature phonologique des unités qu'ils affectent et secondairement par l'environnement syntaxique dans lequel ces unités se rencontrent. Malgré une importante régularité, il existe des exceptions célèbres à ces deux phénomènes qui s'expliquent aisément par la diachronie (ex : le $h$ aspiré dans un haricot, le haricot). Dans cet article, nous traiterons un faisceau d'exceptions moins connues et qui échappent à toute explication diachronique (ex : le orange). Ces exceptions ne sont généralement pas discutées, car traitées en phonologie comme des unités lexicalisées. Même si un certain degré d'arbitrarité peut être envisagé en synchronie, celui-ci pose un problème majeur en diachronie : pourquoi des mots tels que orange ont échappé aux règles de la liaison et de l'élision en français moderne ? Ce problème est d'autant plus saillant qu'une même racine peut donner une forme respectant les règles de la liaison et de l'élision (ex : l'orange) et une forme ne les respectant pas (ex : le orange).

Dans la première section, nous listons ces exceptions inattendues après avoir rappelé la règle générale de la liaison et de l'élision ainsi que ses principaux contrexemples. Dans la seconde section, nous montrons qu'un grand nombre de ces exceptions partagent des propriétés sémantiques et syntaxiques qui expliquent leur caractère divergent. Dans la dernière section, nous traitons plus en détail deux ensembles d'exceptions qui ne semblent pas rentrer dans la même catégorie que les autres.

\section{Liaison et élision}

\subsection{La règle générale}

Du point de vue de la description phonétique du français, le terme de "liaison" renvoie à l'apparition d'une consonne entre deux mots (ex : un vs un $\underline{\sim}$ ami), tandis que le terme d'élision fait référence à la chute d'une voyelle (ex : le v vs l'ami). Du point de vue de la phonologie, la liaison et l'élision désignent plus largement deux alternances de la fin du mot qui affectent certaines consonnes et voyelles lexicales. La liaison est une alternance $\mathrm{C} /$ $\varnothing$ dont le premier membre s'observe devant une voyelle (notée V) tandis que le second membre s'observe devant une consonne (notée C) ou en position finale (notée \#) (1a). L'élision est une alternance $\mathrm{V} / \varnothing$ dont le premier membre s'observe devant une consonne ou en position finale tandis que le second membre s'observe devant une voyelle (1b). Il est à noter que l'élision n'est pas la seule alternance $\mathrm{V} / \varnothing$ du français. Le schwa de le peut également alterner avec $\varnothing$ devant une consonne si le déterminant est précédé d'une voyelle (ex : et l'pote). La différence entre l'élision et cette seconde alternance V/Ø est que la première est obligatoire tandis que la seconde est optionnelle. Il est important de prendre garde à cette différence, car une voyelle qui ne subit pas l'élision peut optionnellement chuter en suivant la loi des trois consonnes de Grammont (1914). 
(1) _ $\mathbf{V}$

a. $\quad u \underline{n} \smile a m i$

petit_inconvénient

gros $\_$ennuis

léger_incident
$-\mathbf{C}$

un pote

petit problème

gros soucis

léger contretemps

le pote

la copine

tu mets

si tu mets
- \#

(prends-en) un

(il est) petit

(il est) gros

(il est) léger b. l'ami

l'amie

t'as mis

s'il met (prends-) $l \underline{e}$

(prends-) la

(mets-)t $\underline{u}$

(avec des) $s \underline{i}$

On peut remarquer que les positions pré-consonantique et finale forment un contexte disjonctif puisque les deux conditionnent de façon identique la non-réalisation d'une consonne (ex : un pote, un) ou la réalisation d'une voyelle (ex : le pote, le). La référence à un contexte disjonctif peut être soustraite à la formulation de la règle si l'on adopte une terminologie syllabique. En effet, les consonnes et la frontière finale sont des objets qui ont pour point commun de ne pouvoir former à eux seuls une syllabe. En termes de traits, ils sont [-syllabique]. En opposition au terme "noyau" (noté N), qui désigne le centre de la syllabe, nous les rassemblerons donc sous le terme de "anti-noyaux" (noté A). À l'aide de ces deux termes, nous pouvons maintenant établir que le premier membre de l'alternance $\mathrm{C} /$ Ø n'apparait que lorsqu'il est suivi d'un noyau (2a), tandis que le premier membre de l'alternance V/Ø n'apparaît que lorsqu'il est suivi d'un anti-noyau (2b).

(2)

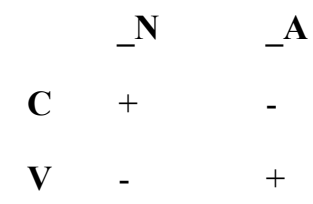

Les membres $\mathrm{C}$ et $\mathrm{V}$ de ces deux alternances, qui font respectivement partie de la catégorie des anti-noyaux et de la catégorie des noyaux, n'apparaissent donc que lorsqu'ils sont suivis d'une unité de catégorie opposée (3).

(3)

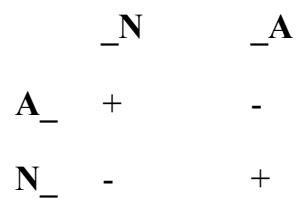

Cette symétrie entre liaison et élision, qui justifie l'emploi dans la suite de l'article du terme chapeau "LE" (Liaison-Élision), est notamment formalisée par Schane (1967) et Tranel (1996) dans un cadre génératif fondé sur des règles pour le premier et des 
contraintes pour le second. Toutefois, cette même symétrie échappe aux développements représentationnels incarnés par la phonologie autosegmentale (Goldsmith 1976). Dans le cadre de cette théorie, Encrevé (1983) propose de voir en la liaison la manifestation d'unités mélodiques et positionnelles flottantes qui, selon les principes de la théorie autosegmentale, ne peuvent se réaliser qu'en s'associant les unes aux autres. Encrevé admet que les consonnes de liaison ne sont associées à aucune position syllabique (4a) et que les mots qui débutent phonétiquement par une voyelle sont introduits par une position antinucléique vide $(4 \mathrm{~b})$. Aucun de ces deux objets ne peut être réalisé sans être associé à l'autre. Pris isolément, ces deux mots sont donc réalisés [pəti] et [ami].

(4)a.

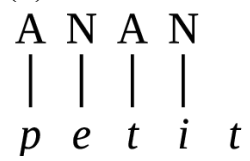

b.

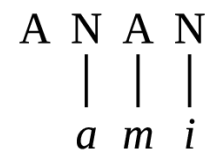

Si la consonne flottante est suivie d'une autre consonne, elle ne peut s'associer à aucune position et reste silencieuse $(5 \mathrm{a})^{\mathrm{ii}}$. Si en revanche elle est suivie d'une voyelle, elle peut s'associer à la position anti-nucléique vide qui précède cette voyelle $(5 \mathrm{~b})$. La position vide et la consonne flottante étant associées, elles se réalisent.

(5)a.

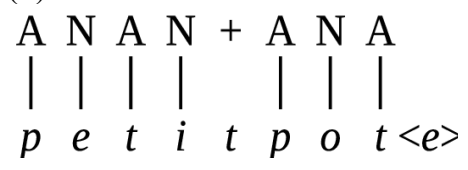

b.

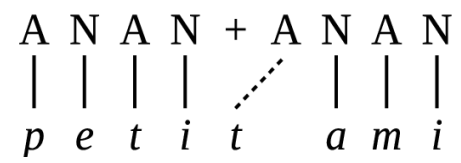

Cette analyse a pour mérite de relier très simplement les principes de la liaison à des types d'unités très largement admis au sein de la phonologie autosegmentale et même en dehors. Toutefois, elle est inapplicable lorsqu'il s'agit de l'élision. Puisque les voyelles qui entrent dans une alternance $\mathrm{V} / \varnothing$ sont réalisées en position finale, il est inapproprié de leur attribuer une représentation flottante (6a). Mais, puisqu'elles sont lexicalement associées, rien de ce que nous avons vu jusqu'ici n'empêche ces voyelles de se réaliser devant d'autres voyelles (c.-à-d. *le ami). Pour rendre compte de l'élision, il faut faire référence à la notion de hiatus. Dans leur article de 1985, Kaye et al. proposent de dériver la tendance qu'ont les langues à éviter les suites de voyelles d'une contrainte née très tôt dans le cadre autosegmental : le Principe de Contour Obligatoire (PCO) (McCarthy 1979, 1986). Ce principe, qui permet de formaliser les processus de dissimilation, stipule que deux unités identiques ne peuvent être adjacentes sur un même niveau autosegmental. Les deux voyelles de la représentation en (6b) ayant chacune une propriété [+vocalique], elles ne peuvent être adjacentes (voir astérisque). La première chute alors pour satisfaire le PCO (voir hachures).

(6)a.

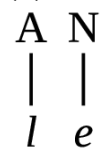

b.

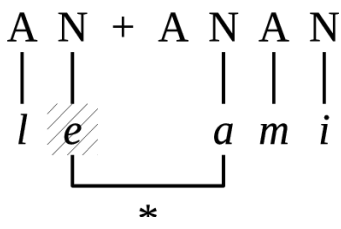


Bien que cette analyse autosegmentale n'unifie pas liaison et élision, on remarque que la généralisation établie plus haut (c.-à-d. le fait qu'une unité n'est réalisée que devant une unité de catégorie opposée) correspond exactement au type de phénomène généré par le PCO. Nous proposons donc une alternative à l'analyse d'Encrevé reposant sur les ingrédients suivant : i. les segments des alternances $\mathrm{C} / \varnothing$ et $\mathrm{V} / \varnothing$ ne sont jamais flottants ; ii. les positions syllabiques identiques ne peuvent jamais se suivre (c.-à-d. elles respectent le PCO, voir Enguehard 2018, 2019) ; et iii. la frontière de phrase notée "\#" se manifeste phonologiquement sous la forme d'une position anti-nucléique ${ }^{\mathrm{iii}}$. Ainsi, lorsqu'une consonne finale précède une voyelle, elle est réalisée puisque la suite d'un anti-noyau et d'un noyau satisfait le PCO (7a). En revanche, si cette même consonne précède une autre consonne (7b) ou une frontière de phrase (7c), on observe une suite de deux anti-noyaux. La consonne finale s'efface donc pour ne pas violer le PCO.

(7)a.

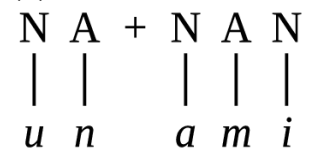

b.

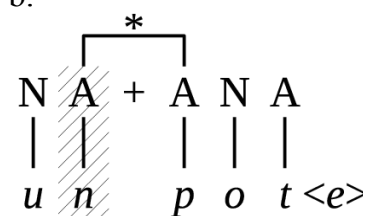

c.

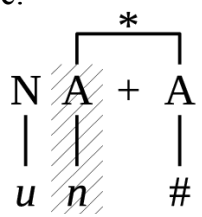

En ce qui concerne l'élision, une voyelle finale suivie d'un noyau viole le PCO et donc chute (8a). En revanche, le PCO est satisfait si cette voyelle est suivie d'une consonne (8b) ou d'une frontière de phrase (8c). La symétrie observée entre la liaison et l'élision est donc respectée par cette analyse.

(8)a.

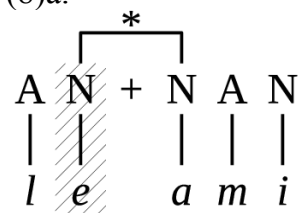

b.

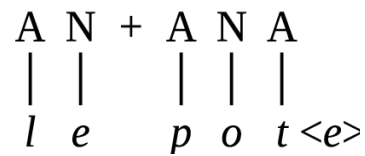

c.

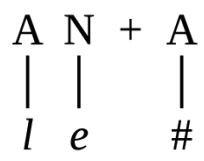

Pour terminer, il est légitime de se demander après cet exposé ce qu'il advient des consonnes et voyelles finales qui n'entrent dans aucune alternance $\mathrm{C} / \varnothing$ ou $\mathrm{V} / \varnothing$ (ex : petite , ami ). Nous admettons ici que ces segments sont suivis d'une position vide, de catégorie opposée à la leur, qui agit comme une zone tampon entre leur position et la position initiale du mot suivant $(9 a, b)$.

(9)a.

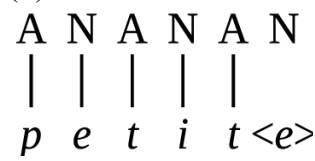

b.

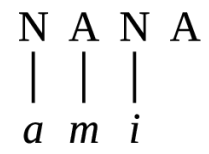

La présence d'une telle position vide est étayée par la diachronie puisque, sauf dans quelques mots d'emprunt (ex : sud), les consonnes finales qui échappent à la liaison sont historiquement suivies d'une voyelle $(10 \mathrm{a})^{\mathrm{iv}}$, et les voyelles qui échappent à l'élision sont historiquement suivies d'une consonne $(10 \mathrm{~b})^{\mathrm{v}}$. Synchroniquement, l'hypothèse d'une 
position vide finale est également appuyée par la présence d'un schwa optionnellement réalisé après les consonnes finales (ex : un[ə]), et d'un contoïde optionnellement réalisé après les voyelles finales (ex : peti $[c]$, où $[\phi])$. L'alternance morphophonologique de certaines racines se terminant par une voyelle (ex : ami $><$ amic-al) peut également indiquer la présence d'une position vide finale dont la réalisation se fait devant un segment de catégorie $\mathrm{N}$ (voyelle), mais pas de catégorie A (consonne ou frontière).

\begin{tabular}{|c|c|c|c|}
\hline (10) & Français & Latin & \\
\hline \multirow[t]{4}{*}{ a. } & une & $u n \underline{a}(m)$ & $c f . u n u(m)>$ un \\
\hline & petite & petila $(m)$ & cf. petilu $(m)>$ petit \\
\hline & grosse & $\operatorname{gross} \underline{a}(m)$ & cf. $\operatorname{grossu}(m)>\operatorname{gros}$ \\
\hline & légère & $\operatorname{leviari\underline {a}}(m)$ & cf. leviariu $(m)>$ léger \\
\hline \multirow[t]{4}{*}{ b. } & $a m i$ & $\operatorname{amic} u(m)$ & cf. si $>s i / s$ \\
\hline & тепи & $\operatorname{minu} \underline{t} u(m)$ & $c f . t u>t u / t$ \\
\hline & feu & foc $u(m)$ & cf. $i l l u(m)>l e / l '$ \\
\hline & $c ̧ a$ & ecce + illa $\underline{c}$ & cf. illa $>$ la/l' \\
\hline
\end{tabular}

Pour résumer, nous pouvons expliquer la symétrie entre liaison et élision à partir d'un principe général qui vaut aussi bien pour la synchronie que pour la diachronie de ces deux phénomènes. Nous abordons maintenant quelques exceptions qui semblent contrevenir à la règle générale.

\subsection{Les exceptions expliquées}

Comme son nom l'indique, la règle générale est valable pour la grande majorité des cas. Toutefois, elle est insuffisante lorsque l'on s'attarde sur certains exemples.

Les mots qui commencent par une semi-voyelle peuvent soit suivre la règle générale (ex : l'oiseau) soit ne pas la suivre (ex : le yaourt). En diachronie, on observe que les semivoyelles qui permettent la LE sont issues de voyelles généralement latines (11a), tandis que les semi-voyelles qui échappent à la LE sont issues de consonnes non-latines (11b).

\section{(11) Français}

a. l'oiseau

l'hiatus

l'huis

b. le wagon $^{v i}$

le yaourt

le whisky

\section{Étymologie}

lat. aucellum

lat. (h)iatus

lat. ostium

ang. wagon

tur. yoğurt

ang. whisky 
En synchronie, Kaye et al. (1984) ont montré que les semi-voyelles du français ont un statut syllabique ambigu : elles se comportent soit comme des voyelles (ex : [w] peut, à l'instar des voyelles, suivre une attaque [tв] dans trois) soit comme des consonnes (ex : [j], à l'instar des consonnes, ne peut suivre une attaque [tь] dans trier). Ils proposent dès lors que le corrélat synchronique de l'observation historique ci-dessus repose sur la syllabicité des semi-voyelles. Celles qui permettent la LE sont associées à un noyau (12a), tandis que celles qui ne permettent pas la LE sont associées à un anti-noyau (12b).

(12)a

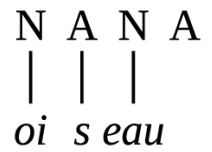

b.

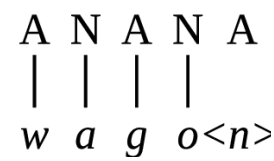

Les notions orthoépiques de $h$ aspiré et $h$ muet permettent de définir une deuxième catégorie d'exceptions connues. Certains mots commençant par un $h$ orthographique échappent à la règle générale (ex : la hache) tandis que d'autres la suivent (ex : l'histoire). Les mots appartenant à la seconde de ces catégories, que l'on dit commencer par un $h$ muet, sont en majorité issus de racines latines à initiale vocalique ou dont le $h$ initial commence à chuter dès la fin de la période républicaine (Zink 1986) (13a). En revanche, les mots commençant par $h$ aspiré sont le plus souvent issus de racines germaniques dont le $h$ initial s'est longtemps maintenu et perdure encore dans certaines régions de France (13b) (ex : normand $[\chi]$ apper vs français happer).

a. l'histoire

l'heure

l'harmonie

b. le houx

le héron

la hache

\section{Étymologie}

lat. (h)istoria

lat. (h)ora

lat. (h)armonia

vha. hulis

vha. heigaro

vha. happia

Comme dans le cas des semi-voyelles, on admet généralement que les mots appartenant à la catégorie $h$ muet commencent par un noyau (14a), tandis que ceux appartenant à la catégorie $h$ aspiré commencent par un anti-noyau vide indépendamment motivé par la diachronie (14b). Dans le second cas, deux hypothèses coexistent : Dell (1973:262) admet que cet anti-noyau est occupé par une occlusive glottale phonologique tandis que Encrevé (1988) ne lui confère aucune substance mélodique. Le choix de l'une ou l'autre de ces hypothèses n'a aucune conséquence sur la suite de notre analyse. 
(14)a.

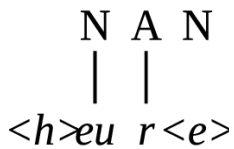

b.

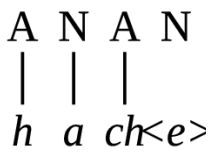

Certains $h$ aspirés ne trouvent en revanche aucune explication diachronique. C'est le cas de héros (issu de la même racine que hérö̈ne qui commence par un $h$ muet) et de hasard (issu de l'espagnol azar). Pour ces rares cas, le principe de généralisation nous permet d'admettre un anti-noyau initial en synchronie, bien que cet anti-noyau ne soit pas confirmé par la diachronie.

Parmi les exceptions connues, on trouve une seconde catégorie de contre-exemples conditionnés par la morphosyntaxe : le cas des noms employés au singulier. Pour Schane (1967) la consonne finale de ces derniers est "obligatoirement" supprimée ; pour Delattre (1966), elles sont également classées parmi les liaisons "interdites" même s'il commente son classement ainsi : "La liaison se fait davantage au pluriel qu'au singulier. Il y a tendance (pas obligation) à marquer le pluriel par une liaison en [z] et le singulier par une absence de liaison". Encrevé (1988), qui s'interroge sur cette catégorie, relève pourtant trois cas de réalisation dans son enquête sur la parole des hommes politiques (ex : le gouvernement_américain, un pays_arabe $)^{\mathrm{vii}}$.

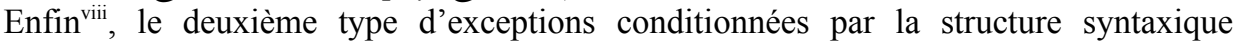
concerne les cas de frontière entre le syntagme nominal sujet et le syntagme verbal. On ne trouvera pas de liaison dans des exemples comme le chat arrive, les amis ont. Même si dans les classements traditionnels (Delattre, 1966), les liaisons après des noms au pluriel étaient considérées comme possibles (facultatives), différentes études de corpus (Durand et Lyche, 2008) tendent aujourd'hui à montrer que la liaison n'est pas faite entre ces deux syntagmes.

Ce dernier type d'exception peut être expliqué par l'analyse présentée plus haut. La frontière de syntagme notée "\#" étant un objet non-syllabique sur le plan phonologique, elle bloque la LE par le biais du PCO (15).
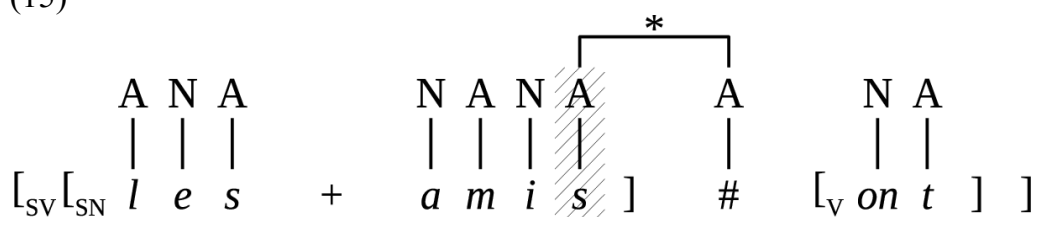

\subsection{Les exceptions inexpliquées}

Il existe enfin un certain nombre d'exceptions qui ne trouvent d'explication ni dans la diachronie ni dans le contexte syntaxique (ex : le un, le $S$, le orange, etc.). Ces exceptions sont balayées par l'analyse phonologique qui, sous prétexte d'une approche synchronique, en fait des phénomènes lexicalisés et leur attribue une occlusive glottale initiale (ex : le $/ \mathrm{R} /$ un) (Scheer, communication personnelle). L'aspect circulaire de cette explication n'est pas tant problématique que le report du problème sur la diachronie. En effet, même si la présence d'une occlusive glottale phonologique (mais phonétiquement absente) dans ces mots est une hypothèse éventuellement acceptable en synchronie, elle est inexplicable en diachronie (ex : pourquoi un /R/ apparaît dans unum $>/ 2 /$ un, mais pas dans uniformem $>$ uniforme). 
Parmi ces exceptions, on trouve les trois noms de nombres commençant par une voyelle (c.-à-d. un, huit, onze) (voir Riegel et al. 1994 : 57). Le mot huit est un peu particulier puisqu'il commence par une semi-voyelle. Toutefois, l'origine de celle-ci étant vocalique (c.-à-d. octo), elle rentre normalement dans la catégorie des mots qui permettent la LE (ex : ostium > l'huis). L'hypothèse d'une occlusive glottale au début de ces mots pose deux problèmes. Premièrement, cette hypothèse fait abstraction du fait qu'il existe une corrélation (même ténue) entre ces exceptions et leur appartenance au paradigme des nombres. Deuxièmement, la racine lexicale de un se retrouve dans l'adjectif transparent unique sans aucune trace d'une quelconque occlusive glottale (ex : un_unique, l'unique).

Les noms de lettres forment un second paradigme de mots qui font tous exception à la LE (ex : le A, le H, le L, etc.). À la différence des nombres, ces exceptions sont optionnelles car on peut aussi bien observer les deux $\smile S$ que les deux $S$. La première de ces formes n'est pas problématique : elle entre dans le cadre de la règle générale et il n'y a rien à en dire. En revanche, la seconde est problématique puisque l'on observe une absence de liaison dans un contexte où elle est pourtant censée être obligatoire. Comme dans le cas précédent, l'hypothèse d'une occlusive glottale lexicalisée en début de ces mots balaie la corrélation entre leur caractère exceptionnel et leur appartenance à un même paradigme.

Les mots représentés orthographiquement par des sigles forment également un sousensemble d'exceptions. Toutefois, à la différence des noms de lettres, seuls les sigles commençant par une consonne graphique font figure d'exception (ex : la SPA, la LSF, le $F M I$, etc.). Ceux qui commencent par une voyelle graphique suivent la règle générale (ex : l'ANPE, l'IVG, l'UE, etc.).

Une couleur (le orange) fait également exception à la LE. Parmi les mots désignant une couleur primaire ou secondaire, c'est le seul qui commence par une voyelle. Les mots désignant une couleur tertiaire suivent la règle générale (ex : l'azur, l'ocre). Étant donné la singularité du mot orange, il est tentant d'admettre l'hypothèse d'une occlusive glottale initiale. Toutefois, le rapport sémantique entre la couleur orange et le fruit orange qui permet la LE (même rapport qu'entre la couleur bleu et le bleu désignant un hématome) implique une alternance arbitraire entre une racine avec occlusive glottale et une racine sans occlusive glottale (le orange vs l'orange).

On trouve également des exceptions dans des paradigmes plus ouverts. C'est le cas des noms propres (le Auchan du quartier, le Onfray d'aujourd'hui, le Orléans d'autrefois, etc.), et des mots mentionnés dans un énoncé à fonction métalinguistique (le "on", le "il", un "ouf" de soulagement, etc) ${ }^{\mathrm{ix}}$.

Enfin, Plénat (1995) note que le verlan des mots monosyllabiques commençant par une consonne font également exception à la LE (ex : le fou > le ouf, le cul $>$ le uc, les seins $>$ les eins, trop pas > trop ap, un joins > un oinj, un chien > un iench, les pieds > les iép). L'hypothèse d'une occlusive glottale appliquée à ces mots est affaiblie par le fait que les mêmes phonologues qui la proposent se donnent pour objectif de dériver les mots du verlan à partir des mots du lexique commun.

\section{Arguments pour une explication syntaxique}

Une explication syntaxique unitaire nous semble possible pour traiter la plupart des cas répertoriés ci-dessus dans la rubrique des exceptions inexpliquées (les emplois autonymiques, les noms de nombres, de lettres, et de couleurs).

Le cas des emplois autonymiques - où le signe est employé non pour renvoyer à son signifié conventionnel mais pour s'autodésigner, comme dans "chat" a quatre lettres, qu'on opposera à le chat a quatre pattes - nous met sur la piste d'une hypothèse explicative générale, applicable dans son principe à l'ensemble des exceptions vues ci-dessus, qui consiste à admettre l'existence d'une position syntaxique vide séparant un élément d'un autre élément avec lequel il est en contiguité de surface. L'absence de la LE (le Bon Usage 
parle de disjonction), au lieu d'être vue comme une exception inexplicable à un phénomène attendu, ne serait que la manifestation en phonologie d'une barrière syntaxique séparant des éléments linéairement contigus.

Symbolisé à l'écrit par une rupture typographique permettant d'isoler le terme mentionné (qui apparaît généralement entre guillemets ou en italiques), et à l'oral par la non-réalisation d'une LE (même lorsqu'elle est obligatoire dans l'usage référentiel ordinaire : le "ami" vs l'ami), l'emploi autonymique d'une unité l'isole de son environnement de surface.

Un nom en mention peut être précédé ou non par un déterminant (Dét), y compris dans les positions syntaxiques qui en exigent un. Nous y reviendrons. Lorsqu'il est précédé par un déterminant, celui-ci, au lieu d'actualiser le référent extralinguistique comme dans la référence ordinaire, actualise des occurrences de son emploi métalinguistique (ex : les "fille" $d u$ texte ne désigne pas une pluralité d'individus filles, mais une pluralité d'occurrences du mot « fille »). Ensuite, sur le plan formel, les jeux d'accord morphologiques montrent clairement des anomalies, inexplicables si on se base uniquement sur les éléments visibles en surface. Quel que soit le genre inhérent du nom, le déterminant qui le précède est en effet systématiquement au masculin (ex : le "filles" de cet exemple). L'accord en nombre semble encore plus déroutant: on peut avoir tout aussi bien $\underline{\text { le }}$ "chevaux" que les "cheval", le premier renvoyant à un emploi unique du terme "chevaux", le second à plusieurs occurrences du terme "cheval". Au lieu de traiter ces cas de nonaccord morphologique de surface comme des exceptions, le traitement le plus pertinent consiste à supposer un terme sous-jacent qui assure ces accords morphologiques (ex : le terme "chevaux" ; les emplois de "cheval"). Enfin, sur le plan syntaxique, tout porte à croire que le terme en mention ne fonctionne jamais comme la véritable tête du syntagme nominal. Il est en effet connu que les traits pertinents - en l'occurrence morphologiques et référentiels - de la tête se trouvent projetés au niveau de tout le syntagme. C'est ainsi qu'on explique par exemple la possibilité d'employer l'adjectif attribut mécontentes dans l'énoncéx Les réalisatrices de ce film à petit budget sont mécontentes de la réaction du public. En vertu de la règle d'accord morphologique entre le sujet et l'attribut, et vu que l'attribut mécontentes est pluriel-féminin, le SN sujet doit lui-même porter ces traits, qu'il ne peut avoir obtenus que par projection depuis la tête du syntagme, en l'occurrence réalisatrices, et non film ou budget. Le même raisonnement s'applique concernant les propriétés référentielles du SN. En vertu des restrictions sélectionnelles, qui conditionnent la combinatoire syntaxique par des traits sémantiques, chaque prédicat spécifie les traits sémantiques que doivent porter ses arguments. Mécontentes ne pouvant se dire que d'un argument spécifié [+Humain], on doit admettre que le SN sujet porte ce même trait, qui ne peut être obtenu que par percolation depuis réalisatrices, qui doit donc être considéré comme la tête de notre SN. Or, le SN d'un nom en mention porte les traits morphologiques du Dét, non ceux du nom présent en surface (ex : les "cheval" du texte sont fréquents ; le "chevaux" de cet exemple illustre ce cas). Sur le plan référentiel, ces SN ne réfèrent pas à des équidés, mais à des mots. En bref, puisque d'une part tout syntagme a une tête, et, d'autre part, les propriétés pertinentes du syntagme lui sont transmises par la tête, il est nécessaire d'admettre l'existence d'une tête vide pour tous les emplois autonymiques. En vertu du Spec-Head Agreement (voir Chomsky 1986), cette tête porte les mêmes traits pertinents que le déterminant. C'est ce mot absent, peu importe lequel, qui, en véritable tête de syntagme, parvient à projeter ses propriétés morphologiques et référentielles au niveau de tout le syntagme. On peut ainsi expliquer les anomalies apparentes au niveau de l'accord morphologique de surface, et générer l'acception métalinguistique du terme en mention. Pour nous, c'est ce même mot sous-jacent qui empêche la LE dans des contextes où elle est pourtant réputée obligatoire, comme dans la suite Dét-N. Systématiquement réalisée dans l'usage habituel (ex : les_enfants ; l'ami), la LE disparaît dans l'emploi autonymique (ex : 
le "ami" de l'exemple précédent; les "enfants" de l'exemple précédent), parce que la contiguïté entre le Dét et le N n'est qu'apparente.

Un terme en mention peut apparaître sans déterminant dans les positions syntaxiques qui exigent habituellement un déterminant, comme la position sujet (ex : "ami" dans cet exemple illustre ce cas). Nous pensons qu'il y a un lien entre cette possibilité qu'a l'autonyme de ne pas être précédé par un Dét et son fonctionnement comme modifieur, non une tête, dans le syntagme où il est employé. En admettant un certain parallélisme entre des syntagmes de type Le mot "ami" et les SN contenant un nom propre dans une structure binominale comme mon frère Jacques, le Président Chirac, qui s'emploient habituellement sans déterminant, on peut attribuer à "ami" la fonction de modifieur du nom (ou épithète dans l'usage grammatical). Or, c'est le traitement que suggère Forsgren (2000) pour les structures binominales de type Le Président Chirac, dans lesquelles "on retrouve l'ordre "inverti", c'est-à-dire que le mot cognitivement caractérisant est sémiotiquement choisi comme nom tête du groupe nominal, et le mot désignant "réellement" celui/celle/ce dont on parle choisi comme épithète, donc comme élément syntaxiquement subordonné." Nous proposons que "ami", en usage autonymique, soit considéré catégoriellement comme un syntagme nominal saturé ( $\mathrm{N}$ ') - condition nécessaire pour qu'il puisse jouer une fonction syntaxique - et fonctionnellement comme modifieur d'une tête nominale dans chacune des trois structures syntaxiques où il peut apparaitre comme autonymique (employé seul, précédé d'un déterminant, ou précédé d'un déterminant suivi d'un terme comme mot par exemple). Dans le schéma ci-dessous, où la fonction modifieur est représentée par une adjonction, ami modifie la tête nominale réalisée lexicalement sous la forme mot. Nous proposons d'étendre cette même structure aux deux autres cas possibles : dans Le "ami" $d u$ texte, la tête nominale n'est pas réalisée lexicalement (on aura dans ce cas sous $\mathrm{N}$ un vide) et dans "Ami" est un mot fréquent dans le texte, on aura un vide aussi bien sous Dét que sous N.

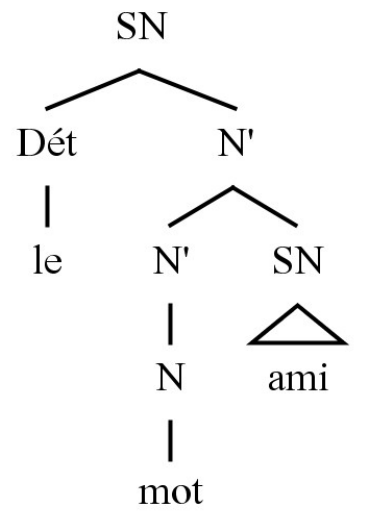

Le cas des noms de lettres figure parmi les exceptions non expliquées dans la mesure où, alors qu'elle est censée être obligatoire, la LE y est simplement possible (ex : le A, le H, le $L$, etc.). Ce cas nous semble en tout point comparable aux emplois autonymiques. On notera d'abord que le référent d'un nom de lettre est essentiellement et souvent la lettre elle-même. S'il peut s'étendre pour désigner le support ou la réalisation particulière du signe (le $S$ rouge), il s'agit d'une désignation métonymique, assez marginale et qui nécessite des contextes pragmatiques particuliers. Comme pour le cas de l'autonymie, un nom de lettre a un usage essentiellement métalinguistique, comme le montrent les conventions typographiques qui le représentent en italique ou entre guillemets. Sur le plan syntaxique, il peut, comme l'emploi autonymique ("ami” a été employé une seule fois dans 
le texte), ne pas être précédé par un Dét (ex : A est la première lettre de l'alphabet; j'aime écrire $S$ ). Mais lorsqu'il est précédé par un Dét, le syntagme nominal porte les mêmes traits de genre et de nombre que le Dét, tandis que le nom de lettre reste invariable en nombre (ex : *des A[z]_illisibles), et, après une certaine hésitation historique (voir le Bon Usage, 2008 : § 480), se présente désormais systématiquement au masculin, comme pour n’importe quel nom en emploi autonymique. Le même raisonnement suivi ci-dessus permet de normaliser l'absence d'accord (ex : tes $A$ ont tendance à pencher), en supposant une tête nominale abstraite qui projette ses propriétés morphologiques et référentielles au niveau du syntagme. Le même schéma syntaxique vu ci-dessus pour les autonymes sera proposé, dans lequel le nom de lettre, considéré comme un $\mathrm{SN}$, joue la fonction de modifieur d'une tête nominale réalisée (Le signe $A$ ) ou non $(A$ ou le $A$ ).

Seul nom de couleur primaire ou secondaire commençant par une voyelle, orange figure parmi les exceptions aux règles générales de la LE, inexplicables en termes phonologiques (voir le orange $=$ couleur $;$ l'orange $=$ le fruit). Orange, dans la suite le orange, correspond soit à un nom (ex : le orange de l'affiche), soit à un adjectif nominalisé (ex : parmi tous ses pulls il a choisi le orange). Ce dernier emploi ne pose pas de difficultés particulières à notre hypothèse générale de l'existence d'une position syntaxique vide séparant le déterminant du nom : l'ellipse est possible parce que le terme omis est restituable. Le terme absent, qui offre un support à l'adjectif de couleur, constitue la véritable tête du syntagme, et parvient à projeter ses traits pertinents, morphologiques et référentiels, au niveau du syntagme dans son ensemble. Dans de toutes ses robes, elle préfère la orange, le $\mathrm{SN}$ la orange est féminin, parce que sa tête est robe; dans de tous ses pulls, il ne met que les orange, le SN les orange renvoie à des pulls. Le raisonnement suivi ci-dessus pour les autonymes et les noms de lettres reste mutatis mutandis valide, et s'étend même aux noms de couleur tertiaire (ex : de tous les cahiers, je choisis le azur et le ocre). Quand il correspond à un authentique nom de couleur (le orange de l'affiche est éclatant), si l'explication par ellipse peut paraître moins intuitive, dans la mesure où il est difficile d'admettre l'existence d'un nom support, il nous semble possible d'établir un lien entre le fait que orange fait partie d'un paradigme restreint (celui des couleurs) et l'existence d'une position syntaxique vide. Nous inspirant des grammaires syntagmatiques (Abeillé, 1993) concernant la structure des traits, où un trait est vu comme un couple attribut-valeur, notre hypothèse consiste à supposer l'existence d'une position syntaxique vide abstraite spécifiant le type d'attribut [attribut couleur] séparant le Dét du nom de couleur. La seule différence qui distingue ces cas de l'emploi nominalisé est que dans ce dernier cas la position vide résulte d'une ellipse, entendue comme effacement d'un élément lexical, restituable contextuellement, alors que dans le cas d'un authentique nom de couleur la position vide est supposée au niveau du système, de la langue : parce qu'elle fait partie d'un paradigme restreint, la valeur occurrente évoque l'attribut sousjacent, qui n'a pas besoin de contexte spécifique pour être restitué. Si on compare $J e$ préfère le gros et Je préfère le orange, on voit que le premier n'est envisageable que dans le cadre d'une ellipse, alors que orange est susceptible d'une double lecture (il évoque soit l'image d'une alternative entre des objets, présents (con)textuellement, se distinguant par la couleur, soit le concept [attribut de couleur]).

La circularité de ce traitement ne semble qu'apparente. Il a l'avantage, d'une part, d'être applicable à l'ensemble des phénomènes observés (les paradigmes des couleurs, des lettres, et, nous le verrons, celui des nombres), et, d'autre part, de proposer une même structure syntaxique pour les $\mathrm{SN}$ examinés ici où on observe une distorsion phonologiquement non explicable aux règles générales de la LE. A la fois parce que le genre d'un SN de couleur, comme pour tous les cas examinés, est toujours au masculin tandis que le pluriel ne semble possible que pour référer à différentes nuances de couleurs, et parce qu'il est susceptible d'entrer dans les trois configurations syntaxiques vues ci-dessus pour les autonymes et les noms de lettres (seul, il est vrai, assez rarement ${ }^{\mathrm{xi}}$, précédé d'un déterminant, ou précédé 
d'un Dét et d'un nom, i.e. la couleur orange), nous proposons d'analyser orange comme un syntagme adjectival qui remplit dans les trois configurations la fonction de modifieur : il modifie une tête nominale lexicalement réalisée dans la couleur orange, mais non réalisée dans le orange. Comme affirmé ci-dessus, cette dernière possibilité - une position vide envisagée au niveau du système et ne correspondant pas à une ellipse restituable contextuellement - n'est réalisable que pour les paradigmes restreints, qui permettent de remonter depuis la valeur au type d'attribut :

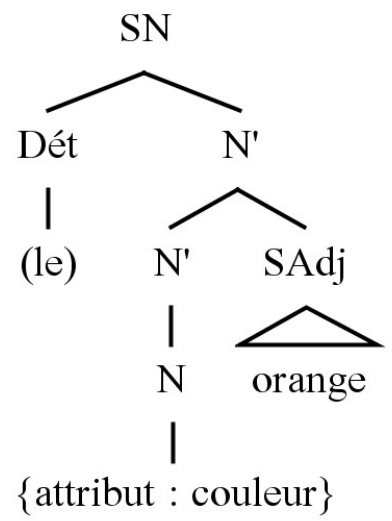

Concernant les noms de nombre (ex : un, huit, onze), lorsqu'ils sont employés comme déterminants, ils ne présentent pas de fonctionnement particulier et aucune distorsion aux règles générales de la LE n'est à signaler (ex : un_enfant, huit_enfants). La même remarque concerne l'emploi pronominal (ex : l'un et l'autre, les_unes et les autres). Les distorsions aux règles générales de la LE ne semblent concerner ces numéraux que dans trois types de contextes syntaxiques : d'abord, les emplois nominaux ou nominalisés (ex : le un, le onze tricolore), ensuite leur emploi, dans un groupe déterminant (Riegel et al. 1994), comme post-déterminants (les adjectifs numéraux cardinaux de la nomenclature grammaticale traditionnelle comme dans les onze joueurs), et, enfin, leur emploi comme modifieurs antéposés au nom, ou adjectifs numéraux ordinaux (le onzième joueur), cas qui sera, faute de place, écarté de notre analyse dans le cadre de ce travail.

En emploi nominalisé ou comme nom, le traitement proposé ci-dessus reste valable mutatis mutandis. En analogie avec les traitements précédents, on admet pour ces syntagmes une structure binominale, composée d'une part d'une tête, pouvant éventuellement être effacée, et d'autre part de la valeur $\mathrm{n}$ du nombre qui remplit la fonction de modifieur de la tête. L'effacement de la tête est possible parce qu'elle est systématiquement restituable. Dans le cas d'une ellipse, la restitution est contextuelle et le déterminant, alors systématiquement présent, s'accorde avec la tête lexicale effacée ( $D e$ toutes ces joueuses, je préfère la huit ; Il a rencontré les onze). Employé comme authentique nom de nombre, $\mathrm{n}$ peut ou non être précédé par un Dét (Onze/Le onze/Le nombre onze est impair). A la fois parce que, lorsqu'il est précédé par un Dét., celui-ci est toujours au singulier masculin, et parce qu'il s'agit d'un paradigme restreint (celui des nombres) permettant de restituer l'attribut depuis la valeur, on peut admettre que l'emploi d'un nombre implique systématiquement une structure sous-jacente de type [(le (nombre)) n], même quand le mot nombre n'est pas réalisé : 


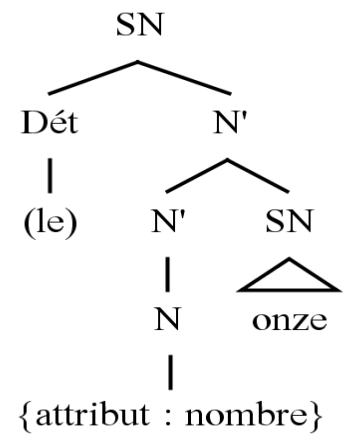

Directement applicable pour les noms de nombre en emploi nominalisé (ex : le un $=$ le numéro un dans les jeux de hasard et les courses ${ }^{\mathrm{xii}}$ par exemple), le traitement proposé pour le nom de nombre pourrait également s'étendre à son emploi comme "adjectif numéral cardinal". Une suite de type Les onze joueurs sera vue comme un syntagme nominal dans lequel le nom de nombre (onze), ayant lui-même le statut initial de syntagme bi-nominal, remplit la fonction de modifieur de la tête joueurs : les onze joueurs $=$ les $[\mathrm{SN}$ nombre $=$ onze] joueurs. On aura ainsi pour ce syntagme une structure de type :

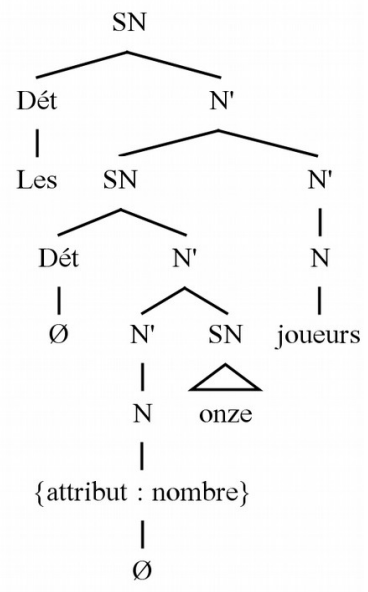

Une telle arborescence - qui pourrait paraître excessive et donner une envie d'élagage reflète la complexité, soulignée depuis longtemps par de nombreux travaux ${ }^{\text {xiii }}$, des quantifieurs numéraux. Elle se distingue d'un traitement syntaxique classique principalement par le statut catégoriel du nom de nombre. Or, il semble que le lien de naturalité entre la fonction de modifieur et la catégorie adjectivale soit à l'origine du traitement du nom de nombre comme adjectif (cardinal), alors qu'il est morphologiquement invariable (voir Soltész 1978).

Cette analyse qui mériterait un examen plus détaillé dans un cadre plus approprié pourrait être étendue à l'emploi du nom de nombre dans le troisième contexte syntaxique, i.e. comme " adjectif ordinal ». Classiquement « construits par dérivation à partir d'une base cardinale : XCARD/CCARD-ième) » (Saulnier \& Amiot, 2014), on peut admettre que le nom de nombre dans son rôle ordinal hérite de la même structure syntaxique supposée cidessus. 
En résumé, ces exceptions à la LE ont pour point commun des propriétés sémantiques et distributionnelles qui laissent supposer la présence d'une tête vide dans leur structure syntaxique. En termes phonologiques, cette tête vide est un objet non-syllabique (c.-à-d. une position anti-nucléique) qui bloque la LE. Comme dans les contre-exemples connus, la LE est empêchée par la présence d'une position vide qui crée une discontinuité structurelle entre deux composants lexicaux.

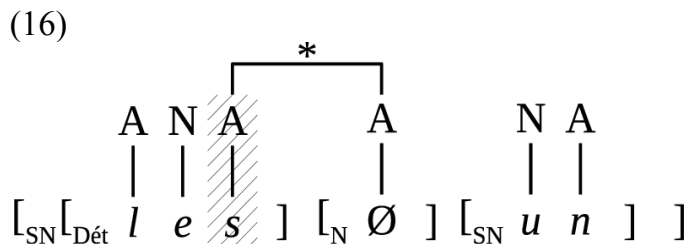

\section{Le cas particulier des sigles et du verlan}

\subsection{Les sigles}

Nous avons montré parmi les exceptions inexpliquées que les sigles se distinguent de l'épellation des lettres quant à la possibilité de respecter la LE. Comment expliquer ce fonctionnement différent dans la mesure où les sigles correspondent, d'un point de vue graphique, à une suite de lettres ? Lettres et sigles se distinguent tout d'abord par le fait que ces derniers n'entrent pas dans un paradigme, contrairement aux lettres (les 26 lettres de notre alphabet), en effet, aucune caractéristique sémantique ou syntaxique ne permet de traiter les sigles comme un ensemble cohérent. Seules finalement la forme graphique (une suite de deux lettres ou plus) et la réalisation phonique (certaines combinaisons rares de phonèmes, ex : /acnpeø/) permettent de caractériser les sigles ${ }^{\text {xiv }}$. Par conséquent, on ne se situe pas ici dans une même catégorie de contre-exemples que ceux évoqués dans la section précédente. Il s'agit de mots qui n'entretiennent aucun lien en synchronie, mais dont l'origine diachronique en fait une classe séparée (comme les mots présentant un $h$ aspiré).

La LE se comportant différemment selon que le sigle commence par une consonne ou une voyelle graphique, il nous faut tout de même rendre compte de cette régularité dans l'émergence des sigles. En suivant Laks (2005) qui "propose d'inscrire l'identité visuelle du mot dans la représentation cognitive" - entendant par identité visuelle notamment les frontières du mot graphique et la consonne finale du mot - nous pouvons expliquer la LE avec les sigles par le fait que la consonne graphique initiale est davantage traitée comme une consonne bloquant la LE que par sa forme phonique qui permettrait la LE, et la voyelle graphique initiale comme une forme permettant la LE, comme toute voyelle initiale. Autrement dit, quand un locuteur évoque le FMI, le traitement cognitif en jeu est identique à celui d'une suite entre un déterminant et un mot à initiale consonantique (ex : un pote, la copine). La même analogie s'opère pour les sigles de type ANPE traités comme des mots commençant par une voyelle. Ce serait donc l'influence de la graphie qui permettrait d'expliquer diachroniquement l'usage particulier de la LE avec les sigles.

\subsection{Le verlan}

Concernant les mots appartenant au lexique du verlan, ils partagent avec les sigles le fait d'être dérivés de mots commençant orthographiquement par une consonne. En diachronie, 
on ne peut donc pas exclure une influence de l'écrit sur leur déviance par rapport à la règle générale de la LE. Certaines formes du verlan relevées par Méla (1991) (ex : le cul > le luc ou le con > le noc) présentent d'ailleurs la caractéristique d'intégrer la consonne finale, non prononcée, de la forme d'origine. Pour expliquer ces formes, on doit faire intervenir la forme graphique du mot d'origine.

En synchronie, en revanche, on ne peut se contenter d'admettre que toutes ces exceptions sont lexicalisées étant donné leur caractère essentiellement oral et productif (ex : le verlan de n'importe quel mot de type CV, par exemple "chat", peut être dérivé sans connaître son orthographe ou sa signification). La théorie autosegmentale rend compte de la plupart des phénomènes de verlan par un procédé métathétique représenté soit par une interversion des positions syllabiques (Rizzolo 2007) soit par une migration de la mélodie d'une position à l'autre (Plénat 1995) ${ }^{\mathrm{xv}}$. Dans le cas du verlan des mots de forme CV, la migration de la mélodie de l'attaque initiale vers l'anti-noyaux qui représente la frontière finale (17a) rend compte de leur caractère exceptionnel. En effet, cette migration laisse une position initiale vide qui bloque la LE comme le ferait une position issue d'un $/ \mathrm{h} /$ étymologique (17b).

(17)a.

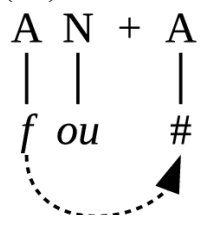

b.

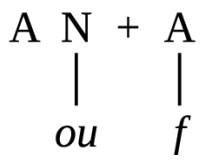

\section{Conclusion}

En conclusion, nous avons montré que certaines exceptions à la LE qui échappent à l'analyse phonologique peuvent s'expliquer par des propriétés sémantiques et syntaxiques qui mettent au jour la présence d'une position vide propageant ses traits à l'ensemble du syntagme. Cette position vide est aisément restituable, soit parce qu'elle est syntaxiquement nécessaire (la tête d'un SN : le X orange), soit parce que l'élément qui subsiste dans le syntagme fait partie d'un paradigme restreint (le paradigme des lettres et des nombres). L'hypothèse d'une position vide en syntaxe bloquant la LE rejoint l'analyse phonologique, proposée par Encrevé (1988) et développée dans cet article, selon laquelle le blocage de la LE devant le $h$ aspiré s'explique par la présence d'une position anti-nucléique vide de toute substance mélodique. En bref, notre étude conclut que l'exception à la LE est avant tout une affaire de discontinuité, aussi bien sur les plans phonologique que syntaxique.

\section{Références bibliographiques}

Abeillé, Anne. 1993. Les Nouvelles syntaxes : grammaires d'unification et analyse du français. Paris : Armand Colin

Borillo, Andrée, Soublin Françoise et Tamine, Joëlle. 1974. Exercices de syntaxe transformationnelle du français. Paris: Armand Colin.

Chomsky, Noam. 1986. Barriers. Linguistic Inquiry Monograph 13. Cambridge, Mass. : MIT Press.

Delattre, Pierre. 1966. Studies in french and comparative phonetics: selected papers in French and English. The Hague, London, Paris: Mouton \& Co.

Dell, François. 1973. Les règles et les sons : Introduction à la phonologie générative. Paris: Hermann.

Durand, Jacques et Lyche, Chantal. 2008. « French Liaison in the Light of Corpus Data ». Journal of French Language Studies. 18 (1): 33-66. 
Encrevé, Pierre. 1983. «La liaison sans enchainement ». Actes de la recherche en sciences sociales 46: 39-66.

-1988. La Liaison avec et sans enchaînement, Phonologie tridimensionnelle et usage du français. Paris: Éditions du seuil.

Enguehard, Guillaume. 2019. " Branching Onsets and the OCP ». in: J. Szpyra-Kozłowska \& M. Radomski (eds), Phonetics and Phonology in Action. 33-46.

- 2018. «Strict CV without Government ». Acta Linguistica Academica 65 (1): 29-45.

Forsgren, Mats. 2000. "Apposition, attribut, épithète : même combat prédicatif ? ", Langue française, $125: 30-45$.

Goldsmith, John A. 1976. " Autosegmental Phonology ». PhD dissertation [ms], MIT. http://hdl.handle.net/1721.1/16388.

Grammont, Maurice. 1914. Traité pratique de prononciation française. Paris: Delagrave.

Grevisse, Maurice et Goosse, André. 2008. Le bon usage. Grammaire française. Bruxelles: De Boeck-Duculot.

Kaye, Jonathan, Jean Lowenstamm, François Dell, D Hirst, et Jean-Roger Vergnaud. 1984. « De la syllabicité ». In Forme sonore du langage, 123-59. Paris: Herman.

Laks, Bernard. 2005. «La liaison et l'illusion ». Langages 158: 101-125.

Lowenstamm, Jean. 1999. "The beginning of the Word». In Phonologika 1996: Syllables!?, édité par John R Rennison et Klaus Kühnammer, 153-66. The Hague: Thesus.

McCarthy, John J. 1979. "Formal problems in Semitic Phonology and Morphology ». Ph. D. Dissertation in Linguistics, MIT.

McCarthy, John J. 1986. OCP effects : gemination and antigemination. Linguistic Inquiry 17 ( 2): 207-264.

Méla, Vivienne. 1991. « Le verlan ou le langage du miroir » Langages 101: 73-94.

Milner, Jean-Claude. 1978. De la syntaxe à l'interprétation : quantités, insultes, exclamations. Paris : Éditions du Seuil.

Pagliano, Claudine. 2003. «L'épenthèse consonantique en français. Ce que la syntaxe, la sémantique et la morphologie peuvent faire à la phonologie: parles-en de ta numérotation ?impossible ». $\mathrm{PhD}$ dissertation [ms], Université de Nice.http://sites.unice.fr/scheer/.

Plénat, Marc. 1995. «Une approche prosodique de la morphologie du verlan ». Lingua 95 (1-3): 97-129.

Riegel, Martin, Pellat, Jean-Christophe et Rioul, René. 1994. Grammaire méthodique du français. Paris: PUF.

Rizzolo, Olivier. 2007. «Schwa final en français: présent ou absent? Le témoignage des jeux de langage ». In Actes des 5èmes journées d'études linguistiques, 137-42.

Saulnier, Sophie \& Dany Amiot. 2014. "Cinquième, vingtième: double catégorisation et grammaire de construction", in S. David, S. Leroys \& D. Villoing. Foisonnements morphologiques. Etudes en hommage à Françoise Kerleroux, pp.249-266. 〈hal-01389738〉

Schane, Sanford A. 1967. «L'élision et la liaison en français ». Languages 2 (8): 37-59.

Soltész, Joseph A. (1978). " Nombre grammatical et système du nombre en français », Cahier de linguistique, 7, 89-135.

Tellier, Christine. 1995. Eléments de syntaxe du français. Méthodes d'analyse en grammaire générative. Montréal : Presses de l'Université de Montréal.

Tranel, Bernard. 1996. «French liaison and elision revisited: A unified account within Optimality Theory ». Aspects of Romance linguistics, 433-55.

Zink, Gaston. 1986. Phonétique historique du français. [1996]. Paris: Presses Universitaires de France. 
' Pour rendre cet article plus accessible, nous avons opté pour une transcription orthographique du français. La liaison et l'élision sont respectivement représentés par les symboles “ ¿ ” et ", “.

ii Les symboles entre chevrons représentent des unités orthographiques qui ne correspondent à aucune réalité phonologique.

iii À ce sujet, voir l'analyse proposée par Lowenstamm (1999) concernant la manifestation phonologique de la frontière initiale du mot.

${ }^{\text {iv }}$ La voyelle latine /a/ chute plus tard que la voyelle /u/ (Zink 1986).

${ }^{\vee}$ La consonne latine $/ \mathrm{m} /$ commence à chuter dès la période archaïque (Zink 1986).

${ }^{v i}$ La consonne initiale est prononcée $[\mathrm{w}]$ dans le nord de la France.

vii Le syntagme accent_aigu est un autre exemple du même type, probablement mieux admis aujourd'hui.

viii Une autre absence de LE est communément admise : après et. Mais ce cas n'est pas à proprement parler une exception à la LE puisque la consonne orthographique finale n'est jamais phonétiquement présente (contrairement au $t$ de gouvernement que l'on retrouve dans gouvernemental). Il ne s'agit que d'un artefact graphique réintroduit vers le XIIè siècle sur modèle du latin et. Les très rares exemples de liaisons faites, donnés par Encrevé (1988:236) étant issus de discours politiques, nous admettons qu'il s'agit d'hypercorrections marginales.

ix Dans ce dernier cas, la LE ne se fait ni avant ni après (ex : le "on" est un pronom vs on est amis ; le "je" est un pronom vs $j$ 'ai un ami).

${ }^{x}$ L'exemple et son analyse sont empruntés à Tellier (1995).

${ }^{\mathrm{xi}}$ On trouve des attestations de type Rouge, bleu et jaune sont les trois couleurs primaires. (https://www.wordreference.com/fres/couleur). On trouve un peu plus facilement des attestations sans déterminant en cas de détachement : rouge, c'est ma couleur préférée. On peut penser que le statut adjectival des noms de couleur est à l'origine de cette résistance à occuper certaines positions syntaxiques.

xii $h$ ttps://www.cnrtl.fr/definition/un

xiii Voir, entre autres, Borillo \& al. (1974) et Milner (1978).

xiv Contrairement aux acronymes qui sont des sigles prononcés comme un mot et non comme une suite de lettres épelées (ex: l'OTAN, l'INSERM, etc.).

${ }^{x v}$ Plutôt qu'une migration des segments, Plénat (1995) admet en fait une réduplication de la racine avec association partielle des segments. L'idée principale, que nous retenons ici, est que les positions du squelette syllabique ne bougent pas. 